\title{
Cue-switch effects do not rely on the same neural systems as task-switch effects
}

\author{
Wouter De Baene • Marcel Brass
}

Published online: 28 August 2011

(C) Psychonomic Society, Inc. 2011

\begin{abstract}
The cued task-switching paradigm is often used to study cognitive control. In this paradigm, people are generally slower and make more errors when switching tasks as compared with repeating the same task. When two cues are mapped to each task, these switch costs could result from a mixture of cue-switch effects (which are thought to reflect cue encoding) and task-switch effects (which are thought to reflect task set preparation). In the behavioral literature, there has been a lively debate on the degree to which cue-switch effects and task-switch effects indeed reflect different phenomena. In the present study, we used fMRI to examine whether and to what extent the neural network underlying task-switch effects is also involved in cue-switch effects. We found task-switch but no cue-switch effects in the frequently observed preparation-related activation in fronto-parietal areas. These results suggest that the fronto-parietal areas displaying preparatory activity in task-switching paradigms are engaged in task preparation but not in cue encoding and that task preparation and cue encoding rely on completely different processes.
\end{abstract}

Keywords Task-switching · Cue-switch · Cue-encoding · Cognitive control

W. De Baene $(\bowtie) \cdot$ M. Brass

Department of Experimental Psychology, Ghent University, Henri Dunantlaan 2,

Ghent 9000, Belgium

e-mail: Wouter.DeBaene@ugent.be

W. De Baene $\cdot$ M. Brass

Ghent Institute for Functional and Metabolic Imaging,

Ghent University,

Ghent, Belgium
In our daily life, we permanently need to adapt our behavior to new task situations. Doing this requires cognitive control processes, which refer to the ability to flexibly adapt one's thoughts and actions in the pursuit of an internal goal. One of the most commonly used experimental paradigms to investigate cognitive control is the task-switching paradigm. When switching between tasks, people are generally slower and less accurate at switching than at repeating tasks (Jersild, 1927). These switch costs are reduced when participants are able to prepare the next task (Meiran, 1996). Over the years, task preparation has become one of the major topics in the taskswitching literature. To examine preparation processes, the task-cuing procedure was proposed (Meiran, 1996), which allowed a closer control over the timing of critical components in task switching. This cuing procedure, however, suffered from a major confound: The cue repeated whenever the task repeated, whereas the cue switched whenever the task switched. Consequently, switch costs could reflect benefits in encoding repeated cues, costs from switching tasks, or both. This confound was addressed by using two different cues per task (Logan \& Bundesen, 2003; Mayr \& Kliegl, 2003), so that a cue change could still result in a task repetition. With this procedure, three types of trial-to-trial transitions are possible: cue-repeat trials, in which task and cue repeat; cue-switch trials, in which the task repeats and the cue switches; and taskswitch trials, in which both task and cue switch. We will refer to the differences between cue-switch and cue-repeat trials as cue-switch effects (reflecting cue encoding; e.g. Brass \& von Cramon, 2004; Logan \& Bundesen, 2003), and to the differences between task-switch and cue-switch trials as task-switch effects (reflecting task set preparation).

The introduction of this 2:1 cue-to-task mapping precipitated a whole controversy on the contribution of 
cue-switching to task preparation. Three different claims have been advanced regarding the degree to which taskswitch and cue-switch effects reflect different phenomena. Logan and Bundesen (2003), in their original publication, reduced task switching to cue switching, attributing all switch costs to cue-related processes. According to Logan and Bundesen (2003), all switch costs could be attributed to cue-related processes - namely a repetition benefit of cue encoding in cue-repeat trials. The opposite view is that cueswitch effects are not related to task-switch effects at all and thus reflect totally different processes. In between these two extreme views, one could argue that cue-switch effects contribute to the switch costs, but that switch costs can not be reduced to cue-switch effects. Mayr and Kliegl (2003), Monsell and Mizon (2006), and Arrington, Logan, \& Schneider (2007), for instance, found that cue-switch effects made up only part of the switch costs and reported substantial task-switch effects.

Although this recent behavioral work might justify the claim that switch costs partly consist of cue-switch effects, these studies have provided only indirect evidence on the level of independence of the underlying processes. First indications on the question whether cue switching and task switching rely on the same or on different processes come from recent EEG work. Jost, Mayr, and Rösler (2008) and Periáñez and Barceló (2009) have provided evidence for distinct mechanisms underlying cue switching and task switching. They showed distinct temporal characteristics and topographical distributions for these effects. However, Periáñez and Barceló (2009) also provided evidence for some overlap between these effects, since their manipulation of cue and task updating both caused significant modulations in the cue-locked P3-amplitude, as well as on other components in the vicinity of the P300 complex.

In the present study, we used functional MRI to test whether cue switching and task switching rely on different or overlapping brain circuits. Several fMRI studies (e.g. McDonald, Cohen, Stenger, \& Carter, 2000; Rushworth, Paus, \& Sipila, 2001; Sohn, Ursu, Anderson, Stenger, \& Carter, 2000) have tried to isolate the neural mechanisms involved in advanced preparation of tasks and reported preparation-related activity in a fronto-parietal network (for a review, see Karayanidis, Jamadar, Ruge, Phillips, Heatcote and Forstmann, 2010). Because they used a 1:1 cue-to-task mapping, however, none of these studies was able to separate cue-switch effects from task-switch effects. Studies that have used a 2:1 cue-to-task mapping have not directly addressed the point to what extent cue switching and task switching rely on overlapping or distinct processes (e.g., Wylie, Javitt, \& Foxe, 2006; see the Discussion section).

The aim of the present study was therefore to examine whether the fronto-parietal areas, which generally show preparatory activity in task-switching paradigms, are pri- marily responsive to cue switching, to task switching, or to both. If cue switching and task switching rely on similar processes, one would expect extensive overlap of both experimental manipulations. If, however, cue switching and task switching rely on different processes, one should expect both manipulations to activate different brain circuits. Finally, if cue switching and task switching share only a part of their underlying processes, these manipulations should result in only partly overlapping areas.

\section{Method}

\section{Participants}

Twenty neurologically normal, right-handed volunteers (eight males, mean age $\pm S D=22.0 \pm 1.8$ ) with (corrected to) normal vision were recruited from Ghent University. They gave written consent before scanning and were paid for participation. One female was excluded for excessive movement. The local ethical committee of the Medical Department of Ghent University approved the study.

\section{Stimuli}

Stimuli (squares with size $=4.5^{\circ}$ ) were presented for $250 \mathrm{~ms}$ at a $60-\mathrm{Hz}$ frame rate on a black screen positioned $120 \mathrm{~cm}$ from the participant. The stimuli were filled with a random texture pattern (50/50 colored and black pixels) moving at a speed of $1.5^{\circ} / \mathrm{s}$. The colored pixels were either red or blue and were luminance matched. The pixels moved up and down or left and right.

\section{Experimental procedure}

On each trial, participants were presented with one of four cues (a square, circle, triangle, or diamond; size $=1.0^{\circ}$ ) for $500 \mathrm{~ms}$. The 2:1 cue-to-task assignments were counterbalanced across participants. After a jittered cue-target interval (CTI), the stimulus was presented for $250 \mathrm{~ms}$, after which the participants had to respond as fast as possible, without sacrificing accuracy. To optimize the separation of cue activity from target activity within a trial, the jittering followed a distribution with pseudologarithmic density: Using steps of $350 \mathrm{~ms}, 50 \%$ of the trials had a CTI ranging from 200 to $1,950 \mathrm{~ms}$. One-third of all trials had a CTI ranging from 2,300 to $4,050 \mathrm{~ms}$, whereas the rest of the trials had a CTI ranging from 4,400 to $6,150 \mathrm{~ms}$. The mean CTI was 2,475 ms.

Participants judged the direction of motion (up-down vs. left-right) of the stimulus in the motion task and the color (red vs. blue) of the colored pixels of the stimulus in the color task, indicating their response with their left or right 
index fingers. The stimulus-response assignments for each task were counterbalanced across participants. After a jittered response-cue interval (RCI; distribution as for the CTI), the next trial started.

Before scanning, all participants were instructed about the two tasks and worked through one practice block for each task separately (32 trials each). The order of these practice blocks was counterbalanced across participants. Afterwards, participants worked through a practice block (48 trials) in which these two tasks were randomly intermixed. In the scanner, participants went through four blocks of 92 trials, each which were equally distributed across the two tasks and randomly intermixed. The task-switch probability was $40 \%$. The other $60 \%$ of the trials were equally distributed across the cue-switch and cue-repeat conditions. Each block started with an instruction screen reminding the participants of the cues and the stimulus-response assignments associated with each task.

\section{Scanning procedure}

Participants were positioned head first and supine in the magnetic bore. Images were collected with a $3 \mathrm{~T}$ Siemens MRI scanner system, using an eight-channel head coil.

First, anatomical images were acquired using a T1weighted 3-D MPRAGE sequence that yielded images with a $1-\mathrm{mm}^{3}$ resolution. Whole brain functional images were collected using a $\mathrm{T} 2 *$-weighted EPI sequence, sensitive to BOLD contrast $(\mathrm{TR}=2000 \mathrm{~ms}, \mathrm{TE}=35 \mathrm{~ms}$, image matrix $=$ $64 \times 64, \mathrm{FOV}=224 \mathrm{~mm}$, flip angle $=80^{\circ}$, slice thickness $=$ $3 \mathrm{~mm}$, distance factor $=17 \%$, voxel size $3.5 \times 3.5 \times$ $3.5 \mathrm{~mm}^{3}, 30$ axial slices).

\section{fMRI Data Preprocessing}

Data processing was performed using the SPM8 software (Wellcome Department of Cognitive Neurology, London, UK). The first four volumes of each run were discarded to minimize T1 relaxation artefacts. Following slice-time correction, realignment, and functional-to-anatomic coregistration, the functional images and anatomic volume were normalized to the Montreal Neurological Institute (MNI) template. Motion parameters were estimated for each session separately. A commonly applied filter of $8 \mathrm{~mm}$ full-width at half maximum and a high-pass filter with a cut-off of $128 \mathrm{~s}$ were used.

Statistical analyses were performed on individual participants' data using the general linear model (GLM) in SPM8. The fMRI time series data were modeled by a series of events: Six different vectors were used, resulting from the factorial combination of task transition (cue repeat, cue switch and task switch) and phase (cue phase vs. target phase). Erroneous trials and trials following errors were modeled separately and were excluded from the analyses.
All of these vectors were convolved with the canonical haemodynamic response function (HRF), as well as with the temporal derivative, and were entered into the regression model (the design matrix). In the model, we also included regressors to account for head motion variance. The statistical parameter estimates were computed separately for each voxel for all columns in the design matrix.

\section{Whole-brain analyses}

Group analyses were performed according to the random effects procedure using the single-participant contrast images (see below) as input. Unless indicated otherwise, group SPMs were generated using a one-sample $t$ test with a voxel threshold of $p<.005$, and a significant effect was reported when the cluster volume exceeded the Monte Carlo simulation-based minimum cluster size of $147 \mathrm{~mm}^{3}$ (42 contiguous voxels), which ensured an overall image-wise false-positive rate of $5 \%$ (corrected $p<.046$; AFNI AlphaSim).

First, we looked for areas reflecting preparatory switchspecific activity by defining a contrast between task-switch and cue-repeat conditions (Condition $\mathrm{C}>$ Condition $\mathrm{A}$; Fig. 1). To find areas reflecting cue encoding, we defined a contrast between cue-switch and cue-repeat conditions (Condition B > Condition A; Fig. 1).

\section{Region of interest analyses}

We first performed a region of interest (ROI) analysis for each of the areas obtained with the task-switch versus cuerepeat contrast to examine whether these areas really reflect preparation. On the basis of the assumption that more preparation leads to faster reaction times (RTs), we subdivided the data for each participant into quartiles, according to the RTs. We reasoned that if the activity in an area really reflects preparation, this activity should decrease with less preparation, thus producing longer RTs. For these analyses, the average beta value in the different quartiles for each ROI was extracted from a $10-\mathrm{mm}$ radius sphere around the peak identified for each area.

Additionally, we performed an ROI analysis for these areas to examine whether cue switching contributed to the

$\begin{array}{lccc} & \text { trial } n & \text { trial } n+1 & \\ \text { Cue repeat } & \text { Task A } & \text { Task A } & \text { Condition A } \\ \text { Cue switch } & \text { Task A } & \text { Task A } & \text { Condition B } \\ \text { Task switch } & \text { Task A } & \text { Task B } & \text { Condition C }\end{array}$

Fig. 1 Definition of the different conditions. In a cue-repeat trial (Condition A, first row), both task and cue repeat. In a cue-switch trial (Condition B, second row), the task repeats, whereas the cue switches. In a task-switch trial (Condition $C$, third row), both task and cue switch 
preparatory switch-specific activity found in these areas. With a similar ROI analysis, we examined whether cue switching contributed to the target activity observed in these areas. For these analyses, the average beta value in cue-repeat, cue-switch and task-switch conditions for each ROI was extracted from a 10-mm radius sphere around the peak identified for each area.

\section{Results}

Behavioural Data

A GLM Repeated Measures ANOVA with RT as a dependent variable and transition (cue repeat, cue switch or task switch) and task (motion or color task) as within-subjects variables
Fig. 2 Activation map averaged across 19 participants mapped onto a standard Colin brain template. Areas displaying stronger activation for taskswitch trials than for cue-repeat trials $(p<.005$, uncorrected, cluster size $=42$ ) are displayed in red
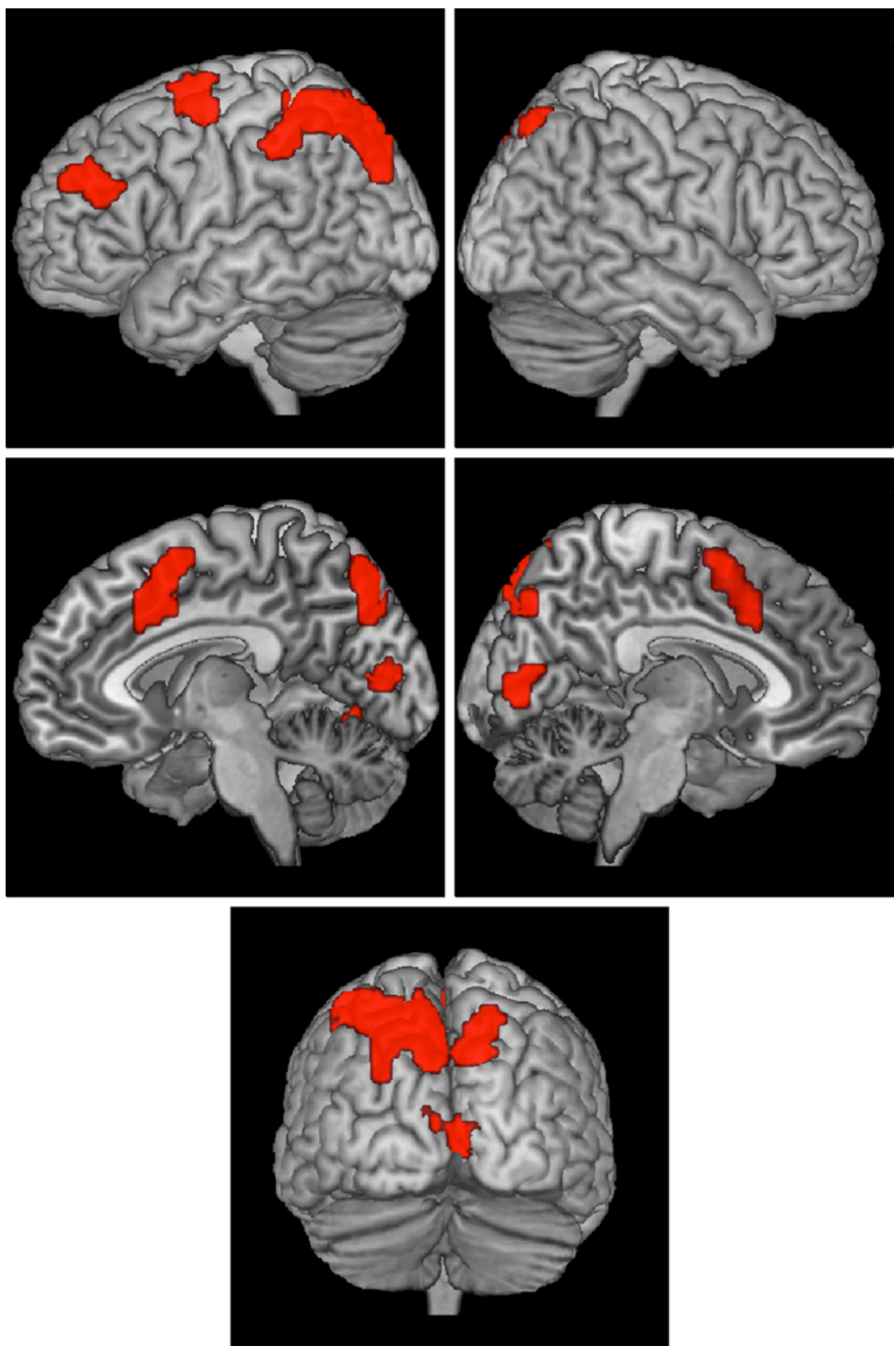
Table 1 Anatomical location and MNI coordinates $(p<.005$, uncorrected) for the task-switch vs. cue-repeat contrast

\begin{tabular}{lllr}
\hline Area & $\begin{array}{l}\text { Peak } \\
\text { Coordinates }\end{array}$ & $z$-score & $\begin{array}{r}\text { Extent } \\
\left(\mathrm{mm}^{3}\right)\end{array}$ \\
\hline Superior parietal lobule & $-8,-77,48$ & 4.79 & 1,617 \\
Superior frontal sulcus & $-32,-4,58$ & 4.10 & 312 \\
Pre-SMA & $-4,7,58$ & 3.87 & 487 \\
Calcarine fissure & $10,-80,6$ & 3.65 & 291 \\
Middle frontal gyrus & $-40,35,27$ & 3.64 & 238 \\
\hline
\end{tabular}

showed a significant main effect of task, $F(1,18)=46.22, p<$ .001 , with generally faster RTs for the color task than for the motion task (784 ms vs. $919 \mathrm{~ms}$ ). The main effect of transition was also significant, $F(2,36)=24.16, p<.001$, which was caused by the significantly slower RTs in the taskswitch condition $(910 \mathrm{~ms})$ as compared with both cue-repeat (809 ms, Fisher LSD: $p<.001$ ) and cue-switch (836 ms, $p<$ $.01)$ conditions. RTs in the cue-repeat condition did not differ from those in the cue-switch condition $(p=.21)$. The interaction between transition and task was not significant, $F(2,36)=1.29, p=.29$.

A similar analysis with proportion of errors per condition as the dependent variable showed no significant effects (lowest $p$ value $>.14$; overall error rate $=5.2 \%$; cue repeat $=5.5 \%$; cue switch $=5.2 \%$; task switch $=5.0 \%$ ).

\section{fMRI Results}

The comparison of task-switch and cue-repeat trials revealed several areas (see Fig. 2 and Table 1) showing switch-specific activity in the preparation phase. These areas were the superior parietal lobule (SPL), superior frontal sulcus (SFS), pre-SMA, and middle frontal gyrus (MFG) in the left hemisphere, and the calcarine fissure in the right hemisphere. Note that using a separate regressor for the fastest (i.e., $200 \mathrm{~ms}$ ) CTI trials (which may be qualitatively distinct from the longer CTI trials, since the possibility to prepare is restricted) or excluding these fastest trials from the model results in highly similar findings.

ROI analyses confirmed that the frontal areas indeed reflected preparation. A repeated measures ANOVA for each of these areas with activity as a dependent variable and quartile as an independent variable showed that the activation in all three frontal areas decreased with longer RTs (all $p \mathrm{~s}<.01$; Fig. 3). No effect of quartile was found for the superior parietal lobule, $F(3,54)=1.42, p=.25$, whereas an opposite effect - that is, an increase in activation with longer RTs, $F(3,54)=4.06, \mathrm{p}<.05$ - was found for the calcarine fissure, an area that is normally not related to task preparation.

Additional ROI analyses confirmed that all areas observed when contrasting the task-switch with the cuerepeat conditions showed significantly higher activation in task-switch than in cue-switch trials and thus reflect taskswitching effects (Fig. 4; Fisher LSD test, all $p s<.01$ ). Furthermore, none of these areas showed cue-switching effects (Fisher LSD, all $p s>$.16). In these preparationrelated areas, no cue-switching effects were found in the target phase (Fisher LSD, all ps $>.11$ ).

When comparing cue-switch and cue-repeat trials, no areas showed cue-switch effects. To find additional support for the lack of cue-switch effects in the areas showing preparatory switch-specific activity, we performed a conjunction analysis
Fig. 3 Region of interest analyses for the areas showing task-switching effects based on reaction times. The average beta value within a $10-\mathrm{mm}$ radius sphere around the peak voxel identified for each area is shown for each quartile

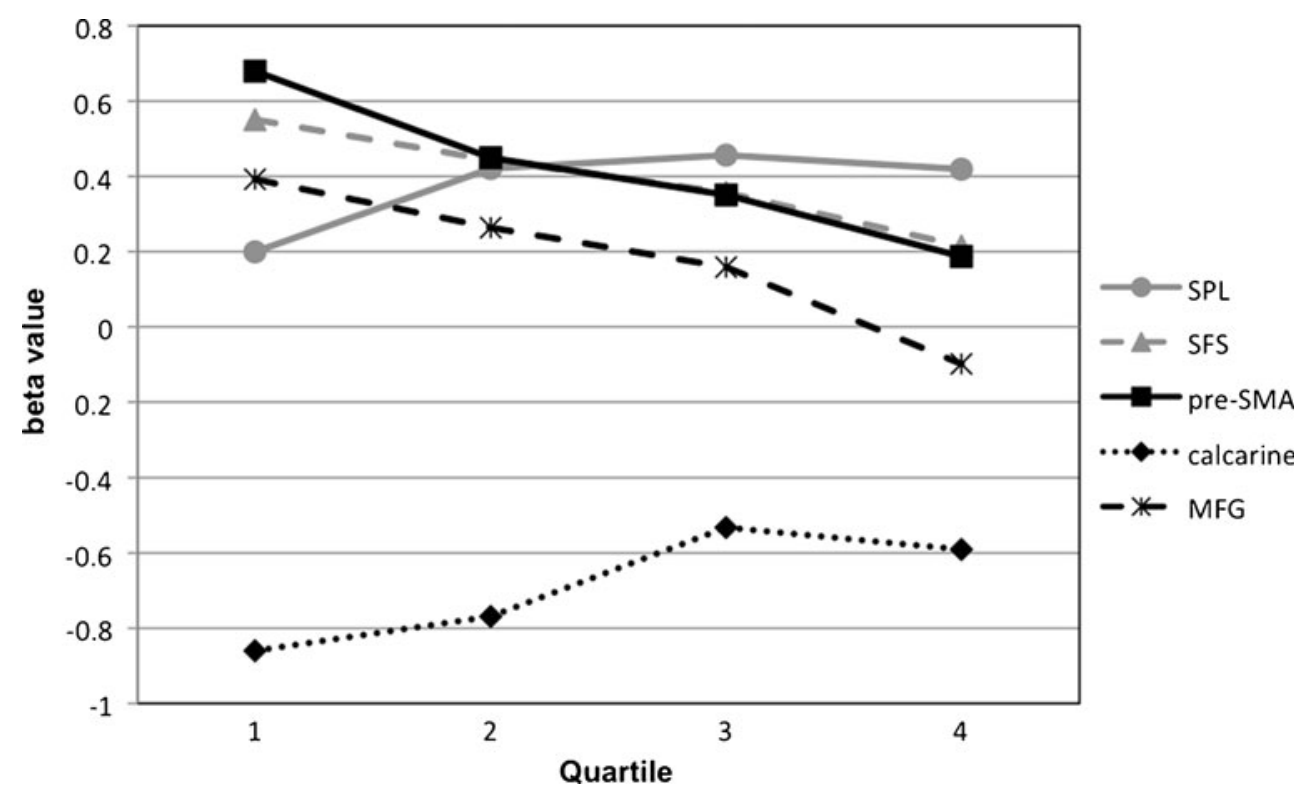


Fig. 4 Region of interest analyses for the areas showing task-switching effects. The average beta value (with $S E$ ) within a 10 -mm radius sphere around the peak voxel identified for each area is shown in the cue-repeat (dark-gray bars), cue-switch (light-gray bars) and task-switch (white bars) conditions

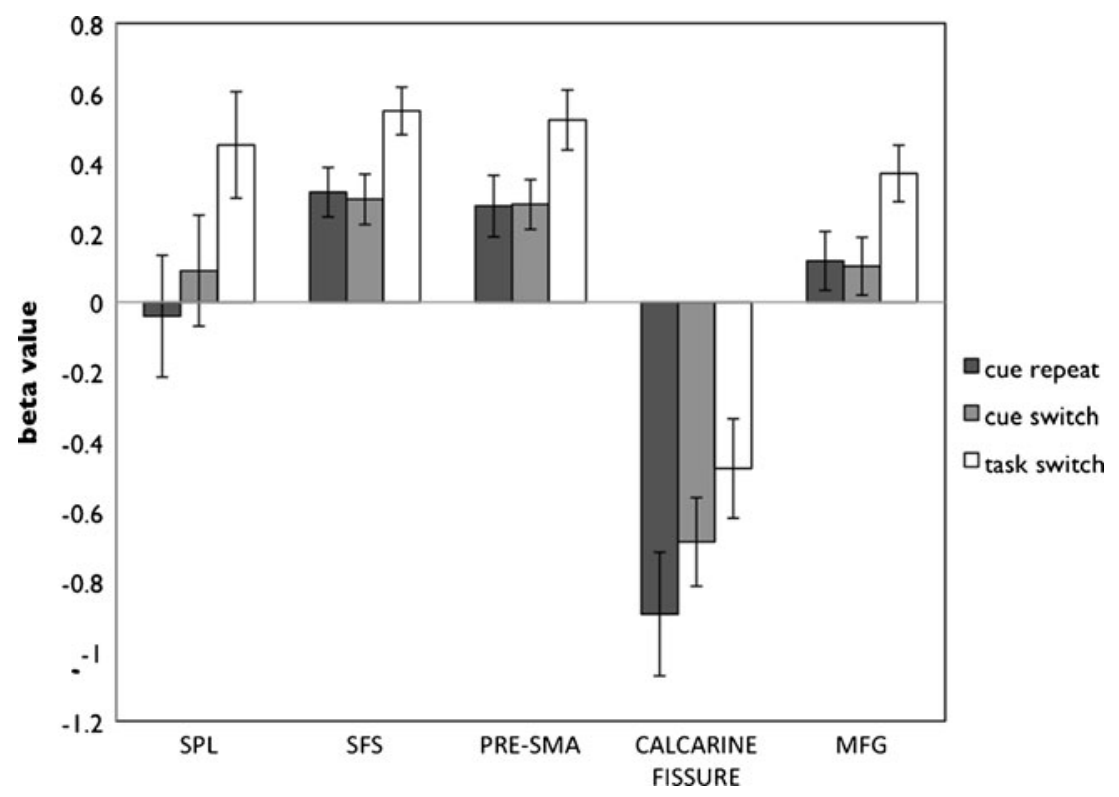

of the two contrasts comparing the task-switch and cue-switch trials to the cue-repeat trials. Using a threshold of $p<.005$ (uncorrected) at voxel level and of $<.05$ at cluster level, no areas were observed, again supporting the finding that the cognitive control areas showing preparatory switch-specific activity do not reflect cue-switch effects.

\section{Discussion}

The present results show that cue switching does not rely on the neural task-switching network. The observed frontoparietal areas, previously described in the literature on task preparation in task switching, did not show any indication for being modulated by cue switching, strongly supporting the assumption that cue switching and task switching rely on completely different processes.

In most fMRI studies on task switching, possible effects of cue switching have been largely neglected. One exception is a study by Brass and von Cramon (2004), who looked at cue-switch effects and suggested that the activation in most preparation-related areas was related to task-set preparation instead of cue encoding. However, since they used a different paradigm, they could not draw strong conclusions about the contribution of cue switching and task switching in the classical task-switching paradigm. Wylie et al. (2006) did use a common task-switching paradigm with a 2:1 cue-to-task mapping with fMRI. Although they did not explicitly examine areas involved in cue encoding, which was not the primary focus of the study, they did report very similar results as in the present study - that is, that no overlap between cue-switching and task-switching areas was observed.
Since our results suggest that preparatory switch-specific activity does not reflect cue encoding, the question remains as to what is exactly represented by the activation difference between switch and repeat trials in these areas. According to the reconfiguration view (e.g., Meiran, 1996; Monsell \& Mizon, 2006; Rogers \& Monsell, 1995), the higher activation in these areas in switch as compared with repeat trials is due to the tuning of the cognitive system for a proper execution of the task when switching tasks. Such reconfiguration processes will normally not be needed when the same task is repeated, since the previous task set is still active. Another possibility is that this activation reflects the retrieval of a symbolic mediator (Logan \& Bundesen, 2004). When arbitrary cues are used (which was the case here), people encode the cue and use it to pull a mediator (e.g., the task name) from memory. This mediator priming account can explain higher activation in switch trials than in repeat trials by the facilitated retrieval of the mediator when the task repeats, since the mediator from the previous trial can still be present in short-term memory. On the basis of previous work (De Baene, Kühn \& Brass 2011), however, we suggest that this activation reflects the representation of a task set. Contrary to the reconfiguration view, the activation difference between switch and repeat trials is not due to higher activation in switch trials but is caused by lower activation in repeat trials as a consequence of task set adaptation. However, to reveal the precise nature of the representations in these areas, further research is necessary.

Activity in the fronto-parietal network observed in the present study has generally been thought to be related to advanced preparation in task switching (for a review, see Karayanidis et al., 2010). Our RT-based region of interest 
analyses indeed support the idea of the co-occurrence of higher activation with higher preparation (as indexed by shorter RTs) in the frontal areas. This pattern was, however, not present for the left superior parietal lobule, for which no proof was found for the link between RT and preparatory activity. Although it is unclear why this pattern was not found for the SPL, the mere observation of preparatory switch-specific activity in this area might already indicate that people have prepared, since switchrepeat activation differences in the left superior posterior parietal cortex were previously observed only for prepared but not for unprepared trials (Jamadar, Hughes, Fulham, Michie \& Karayanidis, 2010).

Note that although our results suggest that there is no neuronal overlap between the processes underlying cue switching and task switching, we did observe a taskswitching area that also showed cue-switching effects in a previous study (De Baene et al., 2011). One possible cause for this discrepancy is that the sensitivity of task-switching areas to show cue-switching effects is dependent on the probability that a cue switch indicates a task switch. When the probability of a task switch given a cue switch is much larger than the probability of a task repeat given a cue switch (e.g., 2 to 1 , as in the previous study), it is possible that people are more inclined to start preparing the other task whenever they encounter a cue switch, which is then reflected in the preparatory activity of some areas. When the ratio between a task switch and a task repeat, given a cue switch, is smaller (e.g., 4 to 3 , as in the present study), people may be less inclined to immediately start preparing the other task whenever the cue switches.

Although the application of a 2:1 cue-to-task mapping is imperative to dissociate cue-switch and task-switch effects in behavioral studies, one might question its use in fMRI studies on task switching on the basis of the present results. The fact that no fronto-parietal areas displaying preparatory activity in task switching are engaged in cue encoding, together with the fact that no areas could be observed that reflect cue encoding, suggest that using a 2:1 cue-to-task mapping will reveal no cognitive processes involved in task preparation that are obscured in a 1:1 mapping. Furthermore, although Schneider and Logan (2011) recently suggested that a 2:1 mapping is a viable solution for the inherent and problematic confound in a 1:1 mapping, they did obtain small but reliable switch cost differences between these mappings. Possibly, these differences reflect quantitative processing dissimilarities. However, as long as it is unclear whether these processing differences between a 1:1 and a 2:1 mapping are not qualitative in nature, we feel that one should opt for the use of a 1:1 mapping in fMRI task-switching studies.

We can conclude that cue-switching does not engage the fronto-parietal cognitive network that is involved in switch- ing between tasks, which questions the added value of the use of a 2:1 mapping in fMRI.

Author Note The present research was made possible by the Research Foundation-Flanders (FWO-Vlaanderen), of which W.D.B. is a postdoctoral research fellow, and was further supported by the Special Research Fund BOF08/GOA/011; BOF06/24JZAP; BOF09/ $\mathrm{PDO} / 058$ of Ghent University.

\section{References}

Arrington, C. M., Logan, G. D., \& Schneider, D. W. (2007). Separating cue encoding from target processing in the explicit task cuing procedure: Are there "true" task switch effects? Journal of Experimental Psychology: Learning, Memory, and Cognition, 33, 484-502.

Brass, M., \& von Cramon, D. Y. (2004). Decomposing components of task preparation with functional MRI. Journal of Cognitive Neuroscience, 16, 609-620.

De Baene, W., Kühn, S., \& Brass, M. (2011). Challenging a decade of brain research on task switching: Brain activation in the taskswitching paradigm reflects adaptation rather than reconfiguration of task sets. Human Brain Mapping. doi: 10.1002/ hbm. 21234

Jamadar, S., Hughes, M., Fulham, W. R., Michie, P. T., \& Karayanidis, F. (2010). The spatial and temporal dynamics of anticipatory preparation and response inhibition in task-switching. NeuroImage, 51, 432-449.

Jersild, A. T. (1927). Mental set and shift. Archives of Psychology, 14, 5-81.

Jost, K., Mayr, U., \& Rösler, F. (2008). Is task switching nothing but cue priming? Evidence from ERPs. Cognitive, Affective, \& Behavioural Neuroscience, 8, 74-84.

Karayanidis, F., Jamadar, S., Ruge, H., Phillips, N., Heatcote, A., \& Forstmann, B. U. (2010). Advance preparation in task-switching: Converging evidence from behavioural, brain activation, and model-based approaches. Frontiers in Psychology. doi:10.3389/ fpsyg. 2010.00025

Logan, G. D., \& Bundesen, C. (2003). Clever homunculus: Is there an endogenous act of control in the explicit task-cuing procedure? Journal of Experimental Psychology: Human Perception and Performance, 29, 575-599.

Logan, G. D., \& Bundesen, C. (2004). Very clever homunculus: Compound stimulus strategies for the explicit task-cuing procedure. Psychonomic Bulletin \& Review, 11, 832-840.

Mayr, U., \& Kliegl, R. (2003). Differential effects of cue changes and task changes on task-selection costs. Journal of Experimental Psychology: Learning, Memory, and Cognition, 29, 362-372.

McDonald, A. W., Cohen, J. D., Stenger, V. A., \& Carter, C. S. (2000). Dissociating the role of the dorsolateral prefrontal and anterior cingulate cortex in cognitive control. Science, 288, 1835-1838.

Meiran, N. (1996). Reconfiguration of processing mode prior to task performance. Journal of Experimental Psychology: Learning, Memory, and Cognition, 22, 1423-1442.

Monsell, S., \& Mizon, G. A. (2006). Can the task-cuing paradigm measure an endogenous task-set reconfiguration process? Journal of Experimental Psychology: Human Perception and Performance, 32, 493-516.

Periáñez, J. A., \& Barceló, F. (2009). Updating sensory versus task representations during task-switching: Insight from cognitive brain potentials in humans. Neuropsychologia, 47, 1160-1172.

Rogers, R. D., \& Monsell, S. (1995). Costs of a predictable switch between simple cognitive tasks. Journal of Experimental Psychology: General, 124, 207-231. 
Rushworth, M. F., Paus, T., \& Sipila, P. K. (2001). Attention systems and the organization of the human parietal cortex. Journal of Neuroscience, 21, 5262-5271.

Schneider, D. W., \& Logan, G. D. (2011). Task-switching performance with 1:1 and 2:1 cue-task mappings: Not so different after all. Journal of Experimental Psychology: Learning, Memory, and Cognition, 37, 405-415
Sohn, M. H., Ursu, S., Anderson, J. R., Stenger, V. A., \& Carter, C. S. (2000). The role of prefrontal cortex and posterior parietal cortex in task switching. Proceedings of the National Academy of Science, 97, 13448-13453.

Wylie, G. R., Javitt, D. C., \& Foxe, J. J. (2006). Jumping the gun: Is effective preparation contingent upon anticipatory activation in task-relevant neural circuitry. Cerebral Cortex, 16, 394-404. 